\title{
Lung water content is not increased in chronic cardiac failure
}

\author{
C S O'Dochartaigh, B Kelly, M S Riley, D P Nicholls
}

Heart 2005;91:1473-1474. doi: 10.1136/hrt.2004.052829

in $\mathrm{n}$ patients with acute heart failure, lung water content is increased leading to pulmonary oedema. ${ }^{1}$ This can be detected clinically and radiologically, and makes a major contribution to the severe dyspnoea characteristic of this condition. In contrast, the lung water content in stable chronic cardiac failure has not been defined, as previous studies all included patients with overt fluid overload in their analysis. $^{2-4}$ We therefore carried out a pilot study with computed tomography (CT) measurement of lung water in patients with stable chronic cardiac failure, as well as some patients with acute heart failure, and compared their scans with scans from patients without evidence of heart failure.

\section{METHODS}

Seventeen patients with chronic cardiac failure (three women) took part in this study. The mean age was 60.4 years (range 4l-79 years), mean height $1.73 \mathrm{~m}$ (1.58$1.97 \mathrm{~m})$, and mean weight $83.3 \mathrm{~kg}(64-103 \mathrm{~kg})$. Three were current smokers and four had never smoked. The cause of the heart failure was ischaemic heart disease (10 patients) or dilated cardiomyopathy (seven). All had at least one previous episode of pulmonary oedema and a mean left ventricular ejection fraction of $27 \%$ (range 15-40\%) measured by echocardiography in nine and by gated blood pool scanning in eight. At the time of the study the patients had been stable with treatment for at least six weeks and were in New York Heart Association (NYHA) functional class II $(\mathrm{n}=9)$ or III $(\mathrm{n}=8)$. Patients with lung disease were excluded on clinical and radiological grounds. Treatment consisted of a loop diuretic (mean dose of furosemide or its equivalent $49 \mathrm{mg}$ daily) in 15, an angiotensin converting enzyme inhibitor in all, a $\beta$ adrenoceptor antagonist in eight, and digoxin in nine. On the day of testing, none had fluid overload on clinical or radiological examination. Dyspnoea was assessed by NYHA class and by their peak achieved oxygen consumption on treadmill exercise testing. We also studied four men aged 62-73 years with severe symptomatic heart failure (NYHA IV). All had clinical evidence of fluid overload on the day of examination (see above) but were able to tolerate lying flat for five minutes in the scanner. Exercise testing was not carried out in this group. All patients gave written informed consent for the scan. The protocol had been approved by the local ethics committee.

Control scans were taken from patients attending the radiology department for non-cardiac reasons, who had undergone CT without contrast enhancement. Eight of the 11 were women and the mean age was 46.5 years (range 1677 years). Four were current smokers. Exercise test results were available for four (table 1). The reasons for scanning were possible metastases from non-lung cancer primary tumours (three); lymphoma treatment follow up (two), and one each of pneumothorax, myeloproliferative disorder, abdominal mass, mediastinal mass, diaphragmatic nodule, and resolved lobar pneumonia. Scans were recorded by a Somatom Plus-S spiral CT scanner (Siemens AG, Erlangen, Germany). Exposures were taken during one second of
Table 1 Lung water content (LWC) and exercise capacity (peak achieved oxygen uptake $\left(\mathrm{PVO}_{2}\right)$ ) in heart failure

\begin{tabular}{llllll}
\hline Group & $\begin{array}{l}\text { NYHA } \\
\text { class }\end{array}$ & LWC (\%) & No & $\begin{array}{l}\mathrm{PVO}_{2} \\
(\mathbf{m l} / \mathbf{m i n} / \mathbf{k g})\end{array}$ & No \\
\hline Controls & NA & $6.2(3.2)$ & 11 & $28.0(1.3)$ & 4 \\
CCF & II & $6.4(1.6)$ & 9 & $21.3(5.2)$ & 8 \\
CCF & III & $7.5(4.2)$ & 8 & $15.0(3.7)$ & 6 \\
AHF & IV & $15.3(7.4)$ & 4 & ND & 0 \\
\hline
\end{tabular}

Data are mean (SD).

AHF, acute heart failure; CCF, chronic cardiac failure; NA, not applicable; ND, not done; NYHA, New York Heart Association.

apnoea at full inspiration at $120 \mathrm{kV}$ and $315 \mathrm{~mA}$. The total radiation dose for each scan was about $5 \mathrm{mSv}$. After a frontal scan of the chest was obtained, two $10 \mathrm{~mm}$ slices were selected, one at the level of the carina and one $2 \mathrm{~cm}$ above the dome of the right hemidiaphragm. Images were analysed on the host computer (MicroVAX II/3200) with manufacturer's software (SOMARIS system). The region of interest was defined by tracing the lung field borders on the computer screen. The appropriate scale range was then set $(-1000$ to -500 Hounsfield units (HU) for air predominant tissue, -500 to $-100 \mathrm{HU}$ for water predominant tissue, and -100 to +3071 HU for solid tissue). The lung water content (percentage) was defined as the area $\left(\mathrm{cm}^{2}\right)$ of water predominant lung $\div$ area of air predominant lung (water to air ratio).

Two investigators performed the procedure above jointly for the first nine scans to ensure consistency of interpretation (CO'D, BK). The same observer then analysed the same test on three separate occasions, in random order, from multiple slices to establish a coefficient of variation for withinobserver, within-test repeatability. Between-test repeatability could not be tested, as this would have required repeated scans. Values are given as mean (SD) and were compared by Student's $t$ test. A p $<0.05$ was accepted as significant. Data from the acute heart failure group were not entered into the analysis in view of the small numbers involved but are included in table 1 to allow comparison.

\section{RESULTS}

The coefficient of variation for estimation of lung water content was acceptable at $6.6 \%$. There was no significant difference in lung water content between controls and patients with NYHA II-III chronic cardiac failure, despite a major difference in peak oxygen consumption (table 1). As expected, those patients with severe chronic cardiac failure and evidence of fluid overload had a much higher lung water content.

\section{DISCUSSION}

In a previous study, the lung water content on CT was increased in patients with evidence of pulmonary venous congestion, ${ }^{2}$ but in those without such evidence, increased lung density was observed in the posterior parts of the lungs only. 
Increased extravascular lung water has also been shown in chronic cardiac failure but many of the patients in these studies had clinical evidence of fluid overload at the time of study. ${ }^{34}$ Despite this limited database, many clinicians continue to believe that dyspnoea in patients with chronic cardiac failure is mainly caused by an increase in lung water content.

We chose CT for the present study because of its convenience and availability. Ethical issues associated with the use of such tests in patients in whom there is no clinical indication for scanning resulted in the small number of patients studied. CT, while non-invasive, is a semiquantitative technique, which measures density and assumes that the zone -500 to $-100 \mathrm{HU}$ is due to water, mainly because water is used as a calibrator. Furthermore, it does not permit the distinction of intravascular and extravascular water. Nevertheless, data from this pilot study were sufficient to suggest that lung water content does not increase greatly in patients with stable chronic cardiac failure, and so changes in lung water content are unlikely to contribute to the exertional dyspnoea characteristic of this condition. ${ }^{5}$

\section{ACKNOWLEDGEMENTS}

We are grateful to Ms A Martin for assistance during the scanning and to Dr M Stevenson for statistical advice.

\section{Authors' affiliations}

C S O'Dochartaigh, D P Nicholls, Department of Medicine, Royal

Victoria Hospital, Belfast, UK

B Kelly, Department of Radiology, Royal Victoria Hospital, Belfast, UK M S Riley, Department of Respiratory Medicine, City Hospital, Belfast, UK

Correspondence to: Professor Paul Nicholls, Royal Victoria Hospital, Belfast BT12 6BA, UK; paul.nicholls@royalhospitals.n-i.nhs.uk

Accepted 21 March 2005

\section{REFERENCES}

1 Szidon JP. Pathophysiology of the congested lung. Cardiol Clin 1989;7:39-48.

2 Kato S, Nakamoto T, lizuka M. Early diagnosis and estimation of pulmonary congestion and edema in patients with left-sided heart diseases from histogram of pulmonary $\mathrm{CT}$ number. Chest 1996; 109:1439-45.

3 Grover M, Slutsky RA, Higgins CB, et al. Extravascular lung water in patients with congestive heart failure: difference between patients with acute and chronic myocardial disease. Radiology 1983;147:659-62.

4 Schober $\mathrm{OH}$, Meyer GJ, Boassaller C, et al. Quantitative determination of regional extravascular lung water and regional blood volume in congestive heart failure. Eur J Nucl Med 1985; 10:17-24.

5 Wasserman K, Zhang Y-Y, Riley MS. Ventilation during exercise in chronic heart failure. Basic Res Cardiol 1996;91(suppl 1):1-11.

\section{IMAGES IN CARDIOLOGY}

\section{PR prolongation in aortic root abscess}

A 47 year old man developed malaise, fever, and a new aortic regurgitation murmur. Staphylococcus aureus was isolated from blood cultures. An echocardiogram showed vegetations on the bicuspid aortic valve with moderate regurgitation. Antibiotic treatment for endocarditis was instituted. Subsequent ECGs evidenced progressive lengthening of the PR interval, from $230 \mathrm{~ms}$ at admission (panel A, upper trace) to $280 \mathrm{~ms}$ two days later (panel A, lower trace). Abscess formation in the aortic root was suspected and confirmed by transoesophageal echocardiography (panel B, arrow).

At surgery, endocarditis with para-aortic abscess formation was confirmed. After debridement and valve replacement, total atrioventricular block ensued for which a DDD

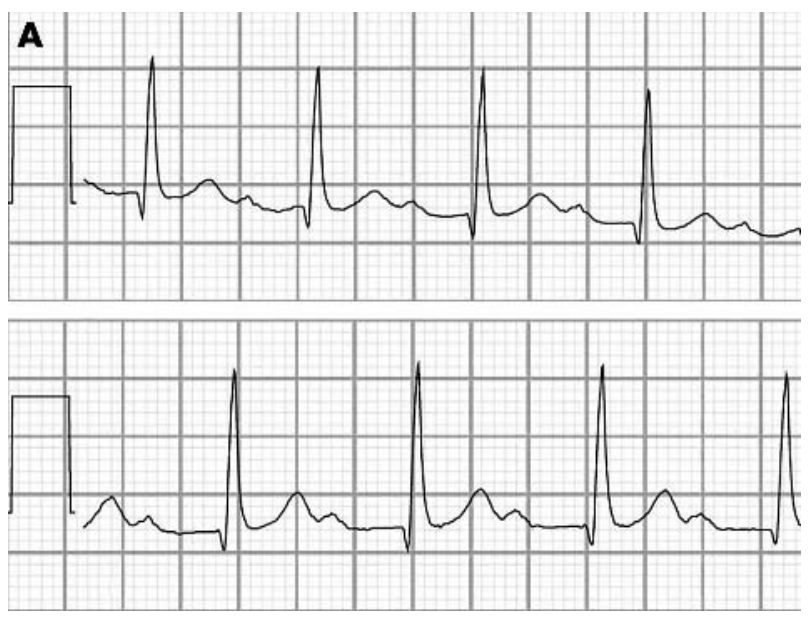

pacemaker was implanted. Thereafter, the patient recovered uneventfully during a six week course of antibiotics.

The ECG is useful for risk stratification of aortic valve endocarditis. Due to the anatomic relation of the aortic root and cardiac conduction system, atrioventricular conduction slowing can occur when infection spreads transmurally across the aortic root. Hence, PR lengthening portends a poor prognosis. Daily electrocardiography should therefore be pursued.

J Lammers

$M$ van Dantzig j.dantzig@chello.nl

There are no conflicts of interest to declare

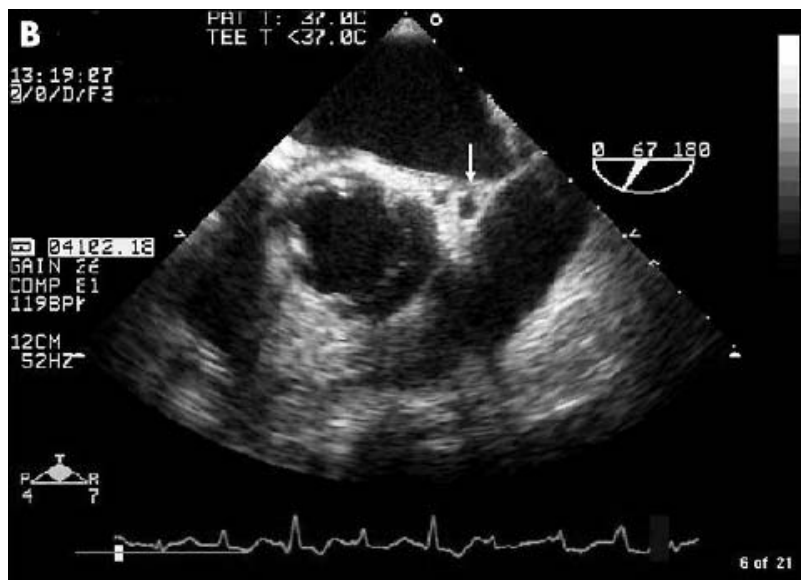

